\title{
Geodetic and Geophysical Applications of High Precision Astrometry
}

\author{
Clark R. Wilson \\ Center for Space Research, Institute for Geophysics, and Department of \\ Geological Sciences, University of Texas Austin, Austin, TX 78712
}

\begin{abstract}
High precision astrometry has a long history of making fundamental contributions to geodesy and geophysics extending centuries into the past, and has provided the foundation for many exciting developments in geophysics in the last several decades. In the 1970s, geodetic VLBI and laser ranging provided the first direct measurement of plate tectonic deformation of the crust. These techniques have also revolutionized the study of Earth rotation by providing sufficient precision to measure climate variability, internal structure, and physical properties. Astrometry will continue to be important in a variety of geophysical problems because of its unique ability to determine Earth orientation relative to a stable celestial frame.
\end{abstract}

\section{Introduction}

Astrometry has made significant contributions to geodesy and geophysics for several centuries, and expectations are that it will continue to do so. The purpose of this paper is to illustrate the value of continued progress through examples of recent contributions to geodesy, the science of measuring Earth's shape, deformation, and gravity field, and to geophysics, which includes the study of Earth's interior structure and climate. Historically, optical astrometric methods were used to measure the shape and moment of inertia of the Earth in the 18th Century and the main features of its rotational variations, including polar motion and rotation rate changes, in the 19th Century. Today geodetic Very Long Baseline Interferometry (VLBI) is probably the most widely recognized form of high precision astrometry, but other techniques, such as laser ranging, are also important. In addition, there is continued interest in historical optical data, some taken centuries ago. Reanalysis of historical data with modern star catalogs provides the long time scale perspective required to study climate change and flow in the mantle.

In the 1970s, the value of high precision astrometry became evident to a broad geophysical community when VLBI and Satellite Laser Ranging (SLR) provided the first direct measurements of deformation of Earth's surface predicted by plate tectonic theories. Plate tectonic rates were deduced from geological data spanning millions of years, and confirmation that contemporary and geological rates agree captured the attention of geologists and geophysicists worldwide. Similarly, improvements in the ability to monitor Earth rotation using VLBI and other methods have revealed a continuum of climate-driven ef- 
fects and brought modern astrometric methods to the attention of the global community of ocean, atmosphere, and climate scientists.

\section{Astrometric contributions to geodesy}

The evolution of space geodesy over the past several decades has brought us to the current era of millimeter-level positioning. All major techniques, including SLR, VLBI, Lunar Laser Ranging (LLR), and the Global Positioning System (GPS) are, in principle, capable of this precision, though each has particular strengths and weaknesses. For example, SLR provides superior measurements of vertical motion relative to Earth's Center of Mass (COM), but cannot distinguish Earth rotation changes from precession of a target satellite's nodal plane. GPS observations are plentiful, but suffer from poor precision in the vertical, and limited ability to separate GPS satellite constellation precession from Earth orientation changes. VLBI provides no fundamental determination of motion relative to the COM, but uniquely measures Earth's orientation relative to an effectively inertial frame formed by the observed radio sources.

\subsection{The International Terrestrial Reference Frame}

The present International Terrestrial Reference Frame (ITRF) describes locations and rates of motion of hundreds of geodetic observatories on an annual basis. With the proliferation of continuous GPS observations, daily time series of site positions are now being produced, but are not completely compatible with the annual solutions for the ITRF. Consequently, there are plans to develop full ITRF solutions (based on all observations) in time series form, which would be more useful to scientific researchers and practical geodesists, and more consistent with Earth rotation determinations already given in time series form. To achieve this goal, temporally and spatially dense GPS observations must be combined with temporally and spatially sparse VLBI, SLR, LLR, and other observations which contribute unique strengths. Combination at the level of original observations is a logical step, replacing the current practice of determining solutions at specific epochs separately for VLBI, SLR, and GPS, and then combining them in some fashion.

\subsection{Earth orientation changes}

Much geophysical information comes directly from observing changes in Earth orientation, normally described in terms of Length of Day (LOD), Polar Motion (PM) and Precession-Nutation (PN). LOD measures the magnitude of the rotation vector, $\mathrm{PM}$ gives changes in position of the rotation axis relative to a terrestrial frame, and $\mathrm{PN}$ describes rotation axis orientation relative to a celestial frame. In practice, an intermediate reference, the Celestial Ephemeris Pole (CEP) is employed in definitions of LOD, PM, and PN (Gross, 1995). Geophysical and geodetic contributions of $\mathrm{PN}$ are covered elsewhere in this volume and the discussion here is restricted to inferences from LOD and PM.

Variations in PM and LOD are forced by large-scale mass redistribution and motion within the Earth system. PM is excited by degree 2, order 1 spherical harmonic components of mass redistribution, and by winds and ocean currents. LOD variations result from degree 2 order 0 spherical harmonic changes in mass 
distribution, and from zonal wind and current variations. Details are provided by Lambeck (1980). Generally at time scales of several years and longer, Earth rotation changes reflect contributions from the mantle and core, while at shorter time scales, variations in the climate system are responsible. Both solid Earth and ocean tides influence LOD and PM over a range of time scales. Reviews of Earth rotation changes and their causes are given by Wahr (1988), Hide and Dickey (1991), Dickey (1995a, 1995b), and Eubanks (1993).

\subsection{Improved tidal models}

Tidal variations in LOD arise from changes in Earth's moment of inertia due to the solid body tides (Yoder et al., 1981), and PM and LOD effects at tidal frequencies are also expected from the tri-axiality of the Earth (Chao et al., 1991). In addition to these solid Earth influences, there are also ocean tide effects on PM and LOD. Understanding these improves the usefulness and precision of geodetic data, which in turn, leads to improved understanding of ocean tides. Changes due to semidiurnal and diurnal tides are of particular interest because these components are large, far from equilibrium, and difficult to model. Regular and intensive VLBI campaigns in the 1980s and early 1990s revealed the existence of tidal PM and LOD changes, and continued observations with VLBI and other techniques now provide a good determination of the signal. At the same time, ocean tide models have improved significantly using satellite radar altimeters (mainly TOPEX), traditional tide gauge data, and a variety of numerical approaches. Ocean tide models now agree reasonably well with observed PM and LOD diurnal and semidiurnal tidal variations (Chao et al., 1996b). Laser ranging to the moon (LLR) is another astrometric observation that measures the ocean tides by constraining energy dissipation rates. Earth-Moon distances have been observed since the first corner cube arrays were placed on the lunar surface in 1969 . The distance increases at a rate of about 3.7 centimeters per year, as a consequence of energy dissipation in the presence of angular momentum conservation within the Earth-Moon system. The lunar semidiurnal tide accounts for most of the dissipation (Wahr, 1988). The LLR measurement of lunar recession nicely complements another form of historical astrometric data, the recorded times of ancient eclipses, which have long been used to estimate tidal energy dissipation rates (Lambeck, 1980).

\section{Contributions to geophysics}

Earth rotation changes provide a global perspective on variations within the solid Earth (crust, mantle and core), and the climate system, (oceans, atmosphere, groundwater, and ice). High precision modern observations of LOD and PM are able to sample both climate and solid Earth effects, and historical optical data are also employed to understand variations at time scales not accessible to modern data. For example, there have been efforts to use optical measurements of LOD from the 19th century as a proxy index for climate change (Salstein and Rosen, 1986), and other applications in geomagnetism are discussed below. 


\subsection{The solid Earth}

The major features of Earth's crust, mantle, and core had been determined by the end of the 1930s using seismic wave travel times. In the absence of convincing additional data, the predominant conceptual model lasting through the 1950s and 1960s was of Earth as a spherically stratified and relatively inert planet. This view changed completely following a succession of geophysical discoveries starting in the 1950s. The activity of Earth's liquid core became evident when it was discovered that the magnetic field has reversed many times. The dynamism of the crust and mantle were confirmed in the 1960s with the determination that the crust moves at many centimeters per year, and consequently about two-thirds of Earth's surface is completely overturned every few hundred million years. More recently, seismic evidence has demonstrated that the thermal convection process responsible for plate tectonics may involve vertical material transport completely through the mantle (Grand, 1994). Thus, most of the Earth below the upper few tens to hundreds of kilometers behaves like a fluid. Understanding the physics of thermal convection requires estimating fluid-related properties of the mantle, especially viscosity. Estimates are derived largely from observations related to Post Glacial Rebound (PGR), the response of the Earth to changes brought about by the end of the last ice age over 10,000 years ago. As North American and Northern European ice sheets melted, sea level rose almost 150 meters, shifting significant loads from the continents to the ocean basins and resulting in flow of mantle material into regions that had been depressed by the ice load. The mantle continues to respond to this load even today, and PGR vertical motion exceeds 1 centimeter per year in some areas (Wu and Peltier, 1984).

VLBI, GPS, and other space geodetic techniques are in principle capable of directly observing PGR crustal deformation, and observations are underway today, mainly using GPS. Unfortunately, horizontal rates due to PGR are very low (millimeters per year), and vertical rates, though larger, present their own measurement challenges. Thus, the main impact of astrometry in estimating mantle viscosity comes from other data types, especially SLR determinations of secular changes in Earth's equatorial bulge (determined by the effect on satellite orbits), and the rate of rotation axis drift, the dominant feature of long-period PM. PGR effects on the equatorial bulge also contribute to long-term changes in LOD, adding to the tidal friction effect discussed earlier. These data provide important global measures of vertical viscosity structure within the mantle, as well as some information concerning history of the ice load. (Peltier, 1986).

At time scales of seconds to about an hour, the mantle behaves as an elastic solid transmitting seismic waves produced by earthquakes. This is quite distinct from the fluid nature of the mantle evident in PGR, and implies a dependency on the duration of an applied load. The term 'visco-elasticity' is used to describe such time-dependent behavior (Ivins and Sammis, 1995). An interest in mantle visco-elasticity at time scales intermediate between PGR and seismic has prompted an effort to observe PM changes produced by large earthquakes. VLBI observations are sufficiently precise to detect the effect predicted for rare very large events, although climate related influences need to be removed first (Chao et al., 1996a). No sufficiently large quake has occurred in recent years, 
but eventually, comparisons between predicted and observed effects will provide new information about mantle visco-elasticity (Soldati and Spada, 1999).

Lunar occultations and other optical data taken over the past several centuries show changes in LOD of many milliseconds over time scales of tens of years, and are confirmed by more recent VLBI observations. These decade scale LOD changes are almost certainly the result of motion in the liquid core, which is also the cause of Earth's main magnetic field. The possibility of using LOD variations to study the core has led to considerable progress in understanding the relationship between magnetic field changes and core fluid motion. Time variations in the main magnetic field spanning several centuries have been determined from historical data, including magnetic observatory and shipboard measurements. At the same time, theories relating magnetic changes with core angular momentum have been developed, allowing magnetic-based predictions of core influence on LOD. Generally, there is reasonable agreement between observed LOD changes and magnetic-based estimates, supporting the general conclusion that the core is responsible for the decade-scale changes. (Jackson $e t$ al., 1993; Jault and Le Mouël, 1989; Hide, 1995). In the future, LOD variations may measure core variability at periods too short to be perceptible in magnetic field fluctuations. In addition the ability to monitor magnetic (hence core momentum) changes and their LOD effects over a range of time scales should provide information on forces coupling the core and mantle.

\subsection{The climate}

An early example of astrometric contributions to climate studies was Jeffreys' (1916) finding that annual PM is due largely to seasonal air and water mass redistribution. Vastly improved precision in PM and LOD data provided by VLBI and other space geodetic methods now reveals climate influences on both LOD and PM over a broad range of time scales, including effects from water and air mass, winds, and ocean current. Comparisons of observed PM and LOD with predictions derived from numerical ocean, atmosphere, and climate models confirm that the continuum of changes in LOD or PM at periods less than a few years are mainly forced by elements of the climate system.

The dominant role of the atmosphere in forcing LOD changes over a broad range of time scales has been known for over two decades. Time series of LOD are highly correlated with axial angular momentum variations of the atmosphere deduced from data assimilating General Circulation Models (GCMs). The small fraction (less than 10\%) of LOD variance not explained by the atmosphere probably comes from the oceans (Marcus et al., 1998). Global sampling inherent in LOD variations nicely complements traditional oceanographic and weather data, and provides useful global measures of the atmosphere and oceans that can be used to test GCM performance.

Although it has long been evident that annual PM is driven by seasonal climate effects, a full understanding of PM at non-seasonal frequencies has only been achieved recently. PM appears to be driven by several separate climate influences. Atmospheric mass redistribution over land appears to explain about half the variance at periods of a year and less, with ocean mass redistribution, winds, and water storage effects contributing the remainder. (Chen et al., 2000). 
At periods of several years and more, terrestrial water storage changes are likely to be the dominant cause of PM. (Chao, 1995; Wilson, 1993)

\subsection{Summary}

Astrometry has a long history of contributing to geodesy and geophysics. Optical data provided the first measures of the shape and rotational variations of the Earth, leading to estimates of the moment of inertia and observations of polar motion, and length of day changes. Modern observations, with VLBI and other space geodetic technologies continue the tradition, determining a millimeter-level terrestrial reference frame, and rotational variations with unprecedented submilli-arc-second precision. In turn, these observations are the basis for modern geodesy, and provide unique geophysical measures of core, mantle, atmosphere, oceans, and hydrology.

Future developments will improve the present situation and broaden the contributions of astrometry to geodesy and geophysics. A new VLBI observing program and data system (Clark et al., 1998) will lead to improved precision, and nearly continuous observations of PN, LOD, and PM. New satellite missions to monitor time variations in the gravity field due to climate change can be supported by high precision PM and LOD time series which also measure effects of climate-related mass redistribution. Advances in data assimilating numerical climate models will provide new insights into the causes of PM and LOD variations. Finally, as astrometric precision improves, new measures of climate can be contemplated. For example, seasonal snow and water loads are known to cause surface displacements of millimeters and larger, levels which are already observable at the best VLBI sites.

\section{References}

Chao, B.F., Dong, D.N., Liu, H.S., and Herring, T.A., 1991, Libration in the Earth's Rotation, Geophys. Res. Lett., 18, 2007-2010.

Chao, B.F., 1995, Anthropogenic impact on global geodynamics due to reservoir water impoundment, Geophys. Res. Lett., 22, 3529-3532.

Chao, B.F., Gross, R.S., and Han Yan-Ben, 1996a, Seismic Excitation of the Polar Motion, 1977-1993, PAGEOPH, 146, 3/4, 407-419.

Chao. B.F., Ray, R.D., Gipson, J.M., Egbert, G.D., and Ma, C., 1996b, Diur$\mathrm{nal} / \mathrm{semidiurnal}$ polar motion excited by oceanic tidal angular momentum, J. Geophys. Res., 101, 20151-20163.

Chen, J.L., Wilson, C.R., Chao, B.F., and Shum, C.K., 2000, Hydrological and Oceanic Excitations to Polar Motion and Length-of-day Variation, Geophys. J. Int., 141, 149-156.

Clark, T.A., Ma, C., Ryan, J.W., Chao, B.F., Gipson, J.M., MacMillan, D.S., Vandenberg, N.R., Eubanks, T.M., and Niell, A.E., 28 April 1998. Earth Rotation Measurement Yields Valuable Information about the Dynamics of the Earth System, EOS, Transactions, American Geophysical Union, 79, 205-216.

Dickey, J.O., 1995a, Earth Rotation, in Global Earth Physics: A Handbook of Physical Constants, American Geophysical Union Reference Shelf 1, 356. 
Dickey, J.O., 1995b, Earth Rotation Variations from Centuries to Hours, Highlights in Astronomy, 10, 17-44.

Eubanks, T.M., 1993, Variations in the Orientation of the Earth, in Contributions of Space Geodesy to Geodynamics, American Geophysical Union Geodynamics Series, 24, Smith and Turcotte Editors, 1-54.

Grand, S.P., 1994, Mantle shear structure beneath the Americas and surrounding oceans, J. Geophys. Res., 99, B6, 11591-11621.

Gross, R.S., 1995, Observations of the Celestial Ephemeris Pole, in I. Appenzeller (ed.), Highlights of Astronomy, 10, 232-236.

Hide, R., 1995, The topographic torque on a bounding surface of a rotating gravitating fluid and the excitation by core motions of decadal fluctuations in Earth's rotation, Geophys. Res. Lett., 22, 961-964.

Hide, R., and Dickey, J.O., 9 August 1991, Earth's Variable Rotation, Science, 353, 629-637.

Ivins, E.R. and Sammis, C.G., 1995, On lateral viscosity contrast in the mantle and the rheology of low-frequency geodynamics, Geophys. J. Int., 123, 305-322.

Jackson, A., Bloxham, J. and Gubbins, D., 1993, Time-Dependent Flow at the Core Surface and Conservation of Angular Momentum in the Coupled Core-Mantle System, Dynamics of Earth's Deep Interior and Earth Rotation, Geophysical Monograph 72, IUGG 12, American Geophysical Union.

Jault, D. and Le Mouël, J.-L., 1991, Exchange of Angular Momentum Between the Core and Mantle, J. Geomag. Geoelect., 43, 111-129.

Jeffreys, H., 1916, Causes contributory to the Annual Variation of Latitude, Mon. Not. R. Astr. Soc., 76, 499.

Lambeck, K., 1980, The Earth's Variable Rotation, Cambridge Univ. Press, London and New York.

Marcus, S.L., Chao, Y., Dickey, J.O., Gegout, P., 11 September 1998, Detection and Modeling of NonTidal Oceanic Effects on Earth's Rotation Rate, Science, 281, 1656-1659.

Peltier, W., 1986, Deglaciation-Induced Vertical Motion of the North American Continent and Transient Lower Mantle Rheology, J. Geophys Res., 91, 9099-9123.

Salstein, D.A. and Rosen, R.D., December 1986, Earth Rotation as a Proxy for Interannual Variability in Atmospheric Circulation, 1860-Present, $J$. Climate and Applied Meteorology, 25, 12, 1870-1877.

Soldati, G. and Spada, G., 1999, Large Earthquakes and Earth rotation: the role of mantle relaxation, Geophys. Res. Lett. 26, 911-914.

Wahr, J.M., 1988, The Earth's Rotation, Ann. Rev. Earth Planet Sci., 16, 231-249.

Wilson, C., 1993, Contributions of Water Mass Redistribution to Polar Motion Excitation, in American Geophysical Union, Geophysical Monograph Series, 24, Contributions of Space Geodesy to Geodynamics: Earth Dynam$i c s$, Smith and Turcotte editors, 77-82. 
Wu, P. and Peltier, W.R., 1984, Pleistocene deglaciation and the Earth's rotation: a new analysis, Geophys. J. R. Astr. Soc., 76, 753-791.

Yoder, C.F., Williams, J.G., and Parke, M.E., 1981, Tidal variations of Earth rotation, J. Geophys. Res., 86, 881-891. 